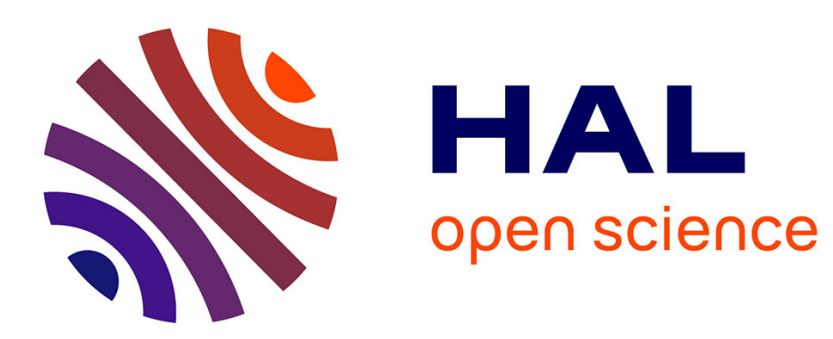

\title{
Mesure et caractérisation de l'attention à l'autre en situation d'interaction stratégique: l'apport de l'économie expérimentale
}

Stéphane Robin

\section{- To cite this version:}

Stéphane Robin. Mesure et caractérisation de l'attention à l'autre en situation d'interaction stratégique: l'apport de l'économie expérimentale. Revue de Philosophie Economique / Review of Economic Philosophy, 2012, 13 (1), pp. 175-191. halshs-00713667

\section{HAL Id: halshs-00713667 \\ https://shs.hal.science/halshs-00713667}

Submitted on 2 Jul 2012

HAL is a multi-disciplinary open access archive for the deposit and dissemination of scientific research documents, whether they are published or not. The documents may come from teaching and research institutions in France or abroad, or from public or private research centers.
L'archive ouverte pluridisciplinaire HAL, est destinée au dépôt et à la diffusion de documents scientifiques de niveau recherche, publiés ou non, émanant des établissements d'enseignement et de recherche français ou étrangers, des laboratoires publics ou privés. 
Mesure et caractérisation de l'attention à l'autre en situation d'interaction stratégique :

l’apport de l'économie expérimentale

Stéphane Robin

Juillet 2012 


\section{GATE Groupe d'Analyse et de Théorie Économique Lyon-St Étienne}

93, chemin des Mouilles 69130 Ecully - France

Tel. +33(0)4 72866060

Fax $+33(0) 472866090$

6, rue Basse des Rives 42023 Saint-Etienne cedex 02 - France

Tel. +33 (0)4 77421960

Fax. +33 (0)4 77421950

Messagerie électronique / Email : gate@gate.cnrs.fr

Téléchargement / Download : http://www.gate.cnrs.fr - Publications / Working Papers 


\title{
Mesure et caractérisation de l'attention à l'autre \\ en situation d'interaction stratégique : \\ l'apport de l'économie expérimentale
}

Stéphane Robin ${ }^{1}$

\begin{abstract}
Résumé :
Dans certaines situations, les comportements réels des individus peuvent apparaître comme des anomalies dans le cadre de la théorie classique de l'agent rationnel. La théorie comportementale vise à expliquer ces anomalies, en particulier celles relatives aux préférences sociales. L'objet de cet article est de montrer comment l'économie expérimentale a permis de mieux mesurer et de mieux caractériser ces préférences. Sur la base d'une revue très sélective d'expériences, nous montrons comment s'est établi un dialogue fructueux entre l'analyse des comportements en laboratoire et la théorie autour de la question de l'attention à l'autre en situation d'interaction stratégique.
\end{abstract}

Mots clefs : Préférence sociale, Equité, Jeu de l'ultimatum, Economie comportementale, Economie expérimentale

\begin{abstract}
:
In some contexts, the actual behavior of individuals can appear as anomalies for a theory of the rational agent. The behavioral theory aims to explain these anomalies, especially those relating to social preferences. The purpose of this paper is to show how experimental economics has provided an accurate measure and further characterization of these preferences. Based on a very selective review of experiences, we show how it was established a fruitful dialogue between the analysis of behavior in laboratory and theory about this question.
\end{abstract}

Keywords: Social preference, Fairness, Ultimatum game, Behavioral economics, Experimental economics

JEL : C92, D03

\footnotetext{
${ }^{1}$ Université de Lyon, Lyon, F-69007, France ; CNRS, GATE Lyon Saint-Etienne, Ecully, F-69130, France.
} 


\section{INTRODUCTION}

La théorie microéconomique vise à expliquer les phénomènes économiques comme l'action d'agents rationnels cherchant à maximiser leur utilité. La question de savoir quel est le contenu d'une telle fonction d'utilité se pose avec acuité pour tout un ensemble de situations. L'absolue indifférence à l'autre, que suppose généralement la théorie, est une source évidente d'anomalies empiriques². L'individu n'est pas indifférent à l'autre, il est animé par des préférences sociales. Il accorde une importance au bien-être de l'autre dans ces décisions. Mais l'identification et la formalisation stricte de la nature de cette attention à l'autre sont difficiles pour deux raisons au moins. La première raison est que l'attention à l'autre est rarement univoque. L'être humain n'est pas, de façon constitutive, altruiste. Il est parfois prêt à agir en fonction des intérêts de l'autre mais il peut tout aussi bien choisir d'adopter un comportement contraire à ces intérêts. Qu'elles soient issues de la sociologie ou de la psychologie, plusieurs propositions sont candidates pour expliquer de tels comportements. L'économiste doit les entendre, les intégrer quand cela est nécessaire, afin d'élaborer des modèles de comportement stricts, non contextuels et susceptibles d'avoir une capacité prédictive la plus forte et la plus large possible. Des travaux de modélisation des fonctions d'utilité comme ceux de Bolton (1991), de Rabin (1993), de Fehr et Schmidt (1999) ou encore de Dufwenberg et Kirchsteiger (2004) vont dans cette direction. Ils se nourrissent des résultats empiriques expérimentaux. La seconde difficulté provient du fait que l'attention à l'autre n'est pas d'une intensité constante d'un individu à l'autre et d'une situation à l'autre. Ainsi, elle pose un problème de pondération quant à l'importance respective des préférences d'ordre individuel et d'ordre social qui caractérisent les agents économiques et de manière concomitante celui de l'incomplétude de l'information quant à l'importance de ces motivations (Meidinger et al., 1999).

Grâce au développement de l'économie expérimentale on a pu, peu à peu, depuis une trentaine d'années, être en mesure de collecter des données empiriques pertinentes et suffisamment fiables pour qu'un dialogue fructueux puisse s'établir entre la théorie microéconomique et les faits. L'objectif de ce programme de recherche vise à construire des fondements théoriques qui soient ancrés dans les comportements effectifs d'acteurs humains : une théorie microéconomique comportementale (Camerer, 2003). Les principaux éléments constitutifs de cette théorie portent sur les questions de l'apprentissage, des règles de décision et des préférences sociales. C'est à cette dernière question que nous nous intéressons dans le présent article.

${ }^{2}$ Par anomalie, nous entendons un résultat empirique qu'il n'est pas possible d'expliquer comme un comportement rationnel à l'intérieur du paradigme théorique, sans recourir à des hypothèses invraisemblables (Thaler, 1988). 
Notre objectif dans cet article est de présenter comment l'économie expérimentale a été mobilisée dans l'analyse et la modélisation des préférences sociales des individus en situation d'interaction stratégique. Il s'agit ici de montrer comment le contrôle et la réplicabilité des conditions d'observations, base de la démarche expérimentale, on permit de prendre la mesure et d'avancer dans la caractérisation des préférences sociales qui orientent les choix individuels. Nous procéderons en deux temps. Dans la section 2, nous montrons comment les observations conduites en laboratoire pour le jeu de l'ultimatum ont conduit à remettre en cause l'hypothèse d'indifférence à l'autre, base de la théorie classique. La section 3 est consacrée aux recherches portant sur la caractérisation des préférences sociales mises en évidence par les expériences du jeu de l'ultimatum³

\section{ULTIMATUMS EN LABORATOIRE : UNE REMISE EN CAUSE DE L'HYPOTHESE D'INDIFFERENCE A L'AUTRE}

Dans le jeu de l'ultimatum deux agents, A et B, doivent partager un profit joint dont le montant est connu. L'agent A propose un partage à $\mathrm{B}$ sous la forme d'un ultimatum. Cet ultimatum détermine la part allant à $\mathrm{A}$ et celle allant à $\mathrm{B}$. Si B accepte la proposition de $\mathrm{A}$, le profit joint est partagé selon les termes de la proposition. Si B refuse cette proposition les gains des deux agents sont nuls.

La situation étudiée est simple et les prédictions de la théorie également. Cette prédiction repose sur trois hypothèses fondamentales relatives aux comportements de A et de B.

- A et $\mathrm{B}$ cherchent à maximiser leurs gains,

- A connaît les motivations de B,

- A est capable de déterminer la proposition qui optimise son gain.

Si B maximise son gain, tout partage lui attribuant un gain non nul sera préféré au rejet de la proposition. Anticipant le comportement de $\mathrm{B}, \mathrm{A}$ fixe le partage de manière à attribuer le gain non nul minimum à $\mathrm{B}$. L'équilibre théorique de l'ultimatum est donc l'issue qui donne à $\mathrm{B}$ la plus petite valeur non nulle que A puisse proposer.

\section{Les comportements observés pour le jeu de l'ultimatum}

$\mathrm{Au}$ début des années 80 , Güth, Schmittberger et Schwarze réalisent la première expérience d'ultimatum et montrent que les comportements observés contredisent la théorie (Güth et al., 1982), ci-après GSS82. Pour cette expérience, des étudiants sont rassemblés dans un même lieu. Le groupe est partagé en deux sous-groupes de taille égale constituant le groupe des sujets A et le groupe des sujets $\mathrm{B}$. Chaque sujet $\mathrm{A}$ fait une proposition pour le partage d'un montant variable ${ }^{4}$. Chaque proposition est attribuée aléatoirement à un sujet $\mathrm{B}$ de sorte qu'aucun des participants ne peut

\footnotetext{
${ }^{3}$ L'objectif de cet article n'est pas d'établir une revue exhaustive de la littérature expérimentale des expériences conçues sur le jeu de l'ultimatum ou sur des jeux apparentés. Pour des revues dans ce domaine, on pourra se reporter à Güth (1995), Roth (1995) ou encore Camerer (2003).

${ }^{4}$ Le montant du profit joint que doit partager chaque sujet $\mathrm{A}$ est fixé de manière aléatoire. Le montant maximum s'élevait à $5 €$ environ.
} 
connaître l'identité de la personne avec laquelle il est associé pour la durée de la session. Chaque sujet B décide alors s'il accepte ou non la proposition qui lui a été attribuée. La rémunération des participants est individuelle et basée sur le résultat du jeu.

On peut résumer les comportements observés en deux propositions relatives au comportement de $\mathrm{A}$ et de B.

A attribue une part à $\mathrm{B}$ qui est supérieure aux prédictions de la théorie. En moyenne, A propose à $\mathrm{B}$ entre $30 \%$ et $40 \%$ du profit joint à partager. La proposition modale correspond majoritairement au partage égalitaire.

B préfère le rejet de la proposition de $\mathrm{A}$ à un partage trop inégalitaire même quand l'acceptation du partage inégalitaire lui procure un gain non nul. B rejette fréquemment les propositions lui attribuant une part inférieur à $20 \%$ du profit joint. D'une manière générale, nous pouvons dire que plus une proposition s'écarte de $50 \%$ plus il est probable que cette proposition soit rejetée par B.

Selon GSS82, il est plausible que A obéisse simultanément à deux motivations : celle de la recherche du gain maximum en tenant compte de la réaction de $\mathrm{B}$ et celle de la recherche d'une solution équitable.

[...] les sujets comptent souvent sur ce qu'ils considèrent comme un résultat juste ou justifié. De plus, l'aspect ultimatum ne peut pas être complètement exploité étant donné que les sujets n'hésitent pas à punir leur opposant qui en demande «trop ». (GSS82)

Les résultats de GSS82 ont suscité un profond intérêt dans la communauté des expérimentateurs. A la suite de cette expérience une série de recherches a été réalisée pour tester la validité et mesurer la robustesse de leurs résultats.

\section{Validité et robustesse des résultats expérimentaux pour le jeu de l'ultimatum}

Quelles sont la validité et la robustesse des résultats obtenus par GSS82 ? Une partie des expériences conduites dans le prolongement du travail de GSS82 porte simultanément sur ces deux questions qui renvoient à des interrogations différentes sur la nature des phénomènes observés dans le jeu de l'ultimatum.

\section{Validité des résultats}

Statuer sur leur validité revient à répondre à la question du degré de contrôle des conditions de réalisation des expériences par le chercheur et à s'interroger sur les éventuels facteurs non pris en compte qui peuvent en perturber le déroulement. Nous nous intéresserons ici à la condition de l'anonymat de la décision. La question de l'anonymat est importante pour des jeux qui font intervenir de façon directe des considérations d'équité dans le choix des participants. L'organisation des sessions de GSS82 est effectuée dans le cadre d'un séminaire d'enseignement en économie. Les conditions dans lesquelles l'expérience est conduite ne peuvent garantir ni l'anonymat des décisions entre participants ni l'anonymat entre les participants et l'expérimentateur. 
L'anonymat participant-participant est la garantie faite à chaque participant qu'il ne connaîtra à aucun moment la personne avec laquelle il est associé pour la durée de l'expérience et qu'inversement la personne avec laquelle il est associé ne pourra à aucun moment connaître son identité. L'absence d'anonymat participant-participant pose le problème de l'influence que peuvent avoir, sur les résultats de l'expérience, les relations qu'entretiennent ou qu'entretiendront les participants en dehors du laboratoire. Plusieurs expériences, dont celle reportée par Roth (1995), montrent que l'absence d'anonymat entre participants conduit à des partages plus égalitaire et de manière consécutive à un une réduction des rejets de proposition. Le fait de connaître l'autre partie, le fait de pouvoir lui parler ou encore de pouvoir la voir influence la manière dont un sujet se comporte en situation de négociation bilatérale. La nature et l'importance de ces influences ne sont pas encore identifiées. En conséquence, la majorité des expériences de négociation sont aujourd'hui conduites à l'aide de protocoles garantissant l'anonymat entre les participants. Si l'absence d'anonymat entre participants à un effet significatif sur les comportements observés dans le jeu de l'ultimatum, il ne constitue pas une explication définitive des comportements observés dans ce jeu.

L'anonymat participant-expérimentateur est la garantie faite au participant qu'à aucun moment l'expérimentateur ne pourra associer une action observée au cours de l'expérience à l'identité de son auteur. Si cet anonymat n'est pas garanti, le participant peut croire que son comportement durant l'expérience aura des effets sur sa relation à venir avec l'expérimentateur et pourra influencer ses chances de participation aux expériences futures. Mais, le participant peut aussi être sensible à l'opinion de l'expérimentateur à son égard. Le participant A de l'ultimatum proposera ainsi un partage équitable pour ne pas paraître avide et égoïste aux yeux de l'expérimentateur. De manière similaire, le participant $\mathrm{B}$ est prêt à refuser un partage inégal pour ne pas avoir l'air cupide et plus motivé par la perspective d'un gain monétaire que par celle de la recherche de la justice et de l'équité. Hoffman, McCabe, Shachat et Smith (1994) et Bolton et Zwick (1995) comparent les résultats obtenus avec garantie de l'anonymat entre les participants et l'expérimentateur et ceux obtenus sans cette garantie pour des jeux proches du jeux de l'ultimatum. Les résultats de ces expériences montrent que l'absence d'anonymat participant-expérimentateur introduit un biais dans les résultats observés. En première analyse, la caractérisation de ce biais semble difficile et dépend en grande partie de la nature de la relation entre l'expérimentateur et les participants. Mais surtout, comme pour l'anonymat entre participants, l'absence d'anonymat participant-expérimentateur ne constitue pas une explication suffisante aux comportements observés dans le jeu de l'ultimatum.

\section{Robustesse des résultats}

L'analyse de la robustesse des résultats obtenus par GSS82 est une condition de leur généralisation. La robustesse de ces résultats a motivé un grand nombre de recherches. Nous nous focaliserons ici sur quelques variables testées : le type de jeu, le montant du profit joint à partager et la culture des sujets expérimentaux. 
La question de la spécificité du jeu de l'ultimatum comme explication possible aux résultats de l'expérience de GSS82 est l'explication avancée par Binmore, Shaked et Sutton (1985). Pour ces auteurs, les comportements observés par GSS82 sont propres au jeu de l'ultimatum. Dans la situation extrême que constitue le jeu de l'ultimatum, le rejet par B d'une proposition est irrémédiable. Binmore et al. appellent « erreur » de B, ce comportement. Ils expliquent que c'est cette crainte de l'erreur de B qui pousse $\mathrm{A}$ à faire des propositions éloignées des prédictions de la théorie. Ainsi, les résultats de GSS82 seraient instables et ne résisteraient pas à une modification des modalités de négociation et en particulier à des situations de négociation en plusieurs étapes. Plusieurs recherches ont visé à étudier cette proposition, chacune de ces recherches montre le faible niveau prédictif de la théorie et conclut sur la nécessaire prise en compte de motivations autres que la simple maximisation des gains individuels. A l'issue d'importants travaux comparant les résultats expérimentaux pour 8 traitements différents ${ }^{5}$, Ochs et Roth résument les résultats des jeux en plusieurs étapes (Ochs et Roth, 1989).

(i) Le négociateur qui débute le jeu bénéficie d'un avantage dans la première période et dans l'ensemble des sous-jeux.

(ii) Contrairement à ce que prédit la théorie, le taux d'actualisation subi par le négociateur qui débute le jeu influence l'issue du jeu.

(iii) Une part non négligeable des premières propositions est rejetée.

(iv) En moyenne, les résultats de la négociation s'écartent de l'équilibre théorique pour se rapprocher du partage égalitaire.

(v) Une part non négligeable des propositions rejetées est suivie d'une contre-proposition moins avantageuse pour celui qui la formule que celle qu'il vient de rejeter.

Les résultats obtenus dans les expériences de négociation montrent donc que les phénomènes mis à jour par le jeu de l'ultimatum ne sont pas propres à ce jeu.

Les comportements observés dans les expériences de GSS82 restent-elles stables lors de l'augmentation du montant du profit joint à partager ? Un individu qui est prêt à perdre $2 €$ en jugeant que cette somme est une part trop faible part de $10 €$, sera-t-il prêt à sacrifier $20 €$ quand la somme à partager sera de $100 €$ ? Les participants aux expériences ne seraient-ils pas plutôt à la recherche d'un gain absolu minimum ? Cette hypothèse est parmi celles qui ont été avancées pour expliquer les résultats de l'ultimatum (Ochs et Roth, 1989). Selon cette hypothèse, il existe un gain minimum en dessous duquel $\mathrm{B}$ est indifférent à l'alternative «obtenir la part proposée par $\mathrm{A}$ » ou «quitter le laboratoire les mains vides ». Le sujet $\mathrm{A}$, anticipant le comportement de $\mathrm{B}$, cherche alors à proposer un partage qui soit pour lui-même le plus profitable tout en garantissant à $\mathrm{B}$ un gain supérieur à ce gain minimum. Pour tester cette hypothèse, Hoffman, McCabe et Smith (1996) analysent les comportements de cinquante couples (A, B) dans le cadre d'un jeu d'ultimatum dans lequel la valeur de la somme à partager s'élève à 100\$. Les résultats de l'expérience sont comparés à ceux obtenus lors

\footnotetext{
5 Pour obtenir ces 8 traitements, Ochs et Roth font varier le nombre de phases de la négociation (négociation en deux phases et négociation en trois phases) et les taux d'actualisation subis par les deux négociateurs.
} 
d'une précédente expérience conduite par les mêmes chercheurs, avec le même protocole mais dans laquelle le montant du profit joint n'était que de $10 \$$. La comparaison des résultats des deux expériences ne montre pas d'effet significatif de la somme à partager sur les résultats. Trois des quatre propositions $(90,10)$ et deux des cinq propositions $(70,30)$ sont rejetées par les participants B. Le gain que $\mathrm{B}$ est prêt à sacrifier pour sanctionner une proposition inéquitable montre que l'estimation du partage par B ne porte pas uniquement sur le gain absolu qu'il obtiendra du partage proposé par A mais porte aussi sur le caractère équitable de ce partage.

Nous abordons maintenant le problème important de l'influence des caractéristiques culturelles des participants aux expériences sur les résultats observés pour le jeu de l'ultimatum. On parle en toute généralité d'effet de population pour désigner cette influence. Dans un jeu d'ultimatum, les étudiants en économie se comportent-ils différemment de ceux qui étudient l'histoire de l'art ? Les résultats de jeux d'ultimatum obtenus aux Etats-Unis sont-ils comparables à ceux obtenus en Europe ?

Les expériences conçues par Roth et al. (1991) ont pour objectif de mesurer les écarts de comportements entre les individus de quatre pays aux cultures très différentes : les Etats-Unis, Israël, le Japon et la Slovénie. Cette mesure de l'effet de population est possible grâce à un travail méthodologique exemplaire dont l'objet est la reproduction à l'identique d'un même protocole dans chacun des quatre pays. Le montant du profit joint à partager est déterminé de manière à assurer une équivalence, en terme de pouvoir d'achat, avec 10\$ aux Etats-Unis. Les résultats de l'expérience montrent que les tendances mises à jour dans l'expérience originale de GSS82 sont robustes à l'effet de population. Il montre aussi qu'il existe des facteurs propres à la population d'origine des participants qui influencent le niveau des partages. Ces facteurs peuvent être la traduction des particularités propres aux membres d'une même communauté ${ }^{6}$.

Carter et Irons établissent l'influence d'un effet population lié à la formation suivie par les étudiants d'un même collège. Certains d'entre eux (les économistes) ont suivi des cours d'économie, les autres (les non économistes) n'ont jamais suivi de cours dans cette discipline. De manière statistiquement significative, les joueurs A économistes formulent des propositions supérieures à celles exprimées par les non économistes. De la même façon, les valeurs de réserve en dessous desquelles il y a rejet des propositions de partage sont supérieures pour les joueurs B économistes. Les participants économistes ont une conception du partage acceptable qui diffère de celle des participants non économistes. Dans un jeu de l'ultimatum, cette différence conduit à des résultats distincts pour les deux populations.

L'expérience originale de GSS82 a motivé la conception d'un grand nombre d'expériences dont l'objet était d'établir la validité et la robustesse des résultats observés au cours de cette première

\footnotetext{
${ }^{6}$ Cependant, il convient d'être extrêmement prudent vis-à-vis d'une explication uniquement culturelle des écarts de comportements entre les différents pays. D'autres variables, indépendantes de la nationalité des sujets, peuvent être avancées, comme la situation des sujets par rapport au service militaire citée par Roth et al. (1991).
} 
expérience sur le jeu de l'ultimatum. Ces expériences ont montré que ces résultats étaient à la fois valides et extrêmement robustes. Les individus participants à un jeu de l'ultimatum ne cherchent pas seulement à maximiser leur gain, ils accordent une grande importance au gain de l'autre et cette attention à l'autre est une connaissance commune des joueurs. La seconde partie de cet article est consacrée à la caractérisation de cette attention à l'autre.

\section{CARACTERISER L'ATTENTION A L'AUTRE}

Parmi les différentes conclusions qui émergent des résultats des expériences d'ultimatum, il apparaît que l'équité joue un rôle important dans le cours de la négociation. Ainsi, le partage égalitaire est le partage le plus fréquemment proposé par A. De la même manière, plus le partage proposé s'écarte du partage égalitaire, plus B tend à rejeter la proposition qui lui est offerte. Les préférences des agents dans le choix des issues du jeu ne sont pas uniquement fondées sur des préférences individuelles, mais aussi sur des préférences sociales, traduisant les sentiments de l'agent économique vis-à-vis de l'autre. La prise en compte des préférences sociales des individus pose de nombreux problèmes. Il est tout d'abord nécessaire de caractériser la nature de ces sentiments. Est-ce de l'altruisme ? Est-ce plutôt l'obéissance à des normes sociales relatives à l'équité ? Est-ce le besoin de se sentir « respecté » par l'autre ? Ensuite, nous devons déterminer comment préférences individuelles et préférences sociales interviennent dans les comportements des agents économiques en situation d'interaction. Comme l'indique Thaler, l'agent dont nous cherchons à expliquer le comportement se situe entre les deux extrêmes que sont l'homo strategicus (gamesman) de la théorie des jeux dont l'unique objectif est de maximiser son gain individuel et l'homo justus (fairman) qui recherche avant tout une solution équitable (Ochs et Roth, 1989 ; Thaler, 1988). Chaque individu compose avec ses préférences sociales et ses préférences individuelles. Le choix d'une stratégie de négociation est le produit du compromis auquel aboutit chaque agent. Ce compromis est sans doute propre à chaque individu et aux circonstances de la négociation.

Dans la troisième partie de cet article, nous procédons en deux étapes. Dans un premier temps, nous proposons trois hypothèses concurrentes relatives aux préférences sociales des agents permettant d'expliquer les comportements observés dans les expériences d'ultimatums. Puis, nous mesurons le degré de pertinence des trois hypothèses présentées à la lumière d'une sélection d'expériences. La méthode expérimentale est ici mobilisée pour mettre en concurrence des propositions d'explications d'un même phénomène.

\section{Hypothèses relatives à la nature des préférences sociales}

Une possibilité de prise en compte des préférences sociales dans la modélisation des préférences des négociateurs passe par la révision de leur fonction d'utilité (Ochs et Roth, 1989). Traditionnellement, l'utilité d'un agent est fonction de son gain personnel. Il est possible de prendre en compte les préférences sociales de l'agent en adjoignant une nouvelle variable relative au gain de 
l'autre. Trois hypothèses qui diffèrent quant à la nature des préférences sociales qui sont prises en compte sont présentées successivement.

\section{Hypothèse de l'altruisme}

C'est l'hypothèse qui est généralement proposée, toutes choses étant égales par ailleurs, un individu accrô̂t son utilité en augmentant le gain monétaire de l'autre. Cette hypothèse permet d'expliquer le comportement du joueur A dans le jeu de l'ultimatum. En revanche, cette hypothèse est en total désaccord avec le comportement de B. En effet, le rejet de la proposition conduit à une réduction simultanée du gain monétaire des deux négociateurs.

\section{Hypothèse de l'équité}

Supposons que les individus obéissent à des normes communes de justice et d'équité. Pour une situation donnée, ces normes indiquent la solution équitable et, toutes choses étant égales par ailleurs, l'utilité de chacun diminue lorsque l'on s'écarte de cette norme. Les modèles de Bolton (1991) et de Fehr et Schmidt (1999) s'inscrivent dans cette logique. Selon cette hypothèse, B rejette la proposition de A quand l'utilité que lui procure le gain monétaire qu'il obtiendrait en acceptant cette proposition ne compense plus la désutilité qu'il ressent en acceptant un partage inégalitaire. Le comportement de A s'explique à la fois par la prise en compte d'un risque de rejet de la part de B et par ses préférences pour une solution équitable. Si cette hypothèse est pertinente, nous pouvons alors dire que l'équité est une motivation en soi pour les deux négociateurs.

\section{Hypothèse de réciprocité}

Supposons maintenant que les agents économiques ne cherchent pas les solutions équitables pour elles mêmes mais désirent être traités de manière juste. Toute action estimée comme injuste dans la situation considérée sera ressentie comme une agression devant faire l'objet de mesure de rétorsion. Les modèles de Rabin (1993) ou encore de Dufwenberg et Kirchsteiger (2004) intègrent cette logique intentionnelle de réciprocité. Dans le cadre du jeu de l'ultimatum, cette hypothèse signifie que B est ainsi prêt à sacrifier la part qui lui est proposée, pour sanctionner le comportement de A quand il juge que ce dernier abuse de sa position à son détriment. Le joueur $\mathrm{A}$, connaissant les motivations de $\mathrm{B}$, cherche alors à formuler une proposition telle que lorsque B met en balance la désutilité attendue d'un partage non équitable et l'utilité procurée par la part qui lui est proposée, il préfère ne pas rejeter la proposition. Comme précédemment, les notions de justice et d'équité ont une place importante dans cette hypothèse. Cependant les logiques sous-jacentes sont radicalement différentes. Dans l'hypothèse précédente, les agents recherchaient les solutions équitables pour elles même. Ici, les individus réagissent à un sentiment d'injustice relatif à leur propre intérêt. Le conflit n'est plus seulement l'expression d'une insatisfaction face une solution inéquitable mais il est aussi le moyen de sanctionner le responsable de cette insatisfaction. 


\section{Comparer les hypothèses explicatives}

Les trois hypothèses exposées ci-dessus devraient permettre d'expliquer tout ou partie des comportements observés dans le jeu de l'ultimatum. La méthode expérimentale permet de mesurer leur degré de pertinence respectif en concevant des expériences adaptées à cet objectif. C'est une sélection de ce type d'expériences conçues autour des formes altérées du jeu de l'ultimatum que nous allons passer en revue. Il ne s'agit pas d'éliminer une hypothèse non pertinente mais de comparer la capacité explicative de chacune des hypothèses pour mieux caractériser la nature des préférences sociales.

Le jeu de l'impunité est une situation intéressante pour comparer nos trois hypothèses. Dans un jeu de l'impunité, A propose un partage du profit joint, B peut refuser le partage proposé mais ce refus n'a d'effet que sur son propre gain. En cas de rejet de la proposition, le gain de A reste celui exprimé dans la proposition tandis que B obtient un gain nul. Ainsi A n'a plus à craindre le rejet de sa proposition. Son choix, dans une telle situation, reflète ses intentions véritables vis-à-vis de B et exprime l'importance qu'il accorde aux considérations de justice et d'équité. Le jeu de l'impunité permet aussi de faire la distinction, pour le comportement de B, entre l'expression d'un désaccord et la volonté de punir l'auteur d'une décision injuste. Les conclusions auxquelles aboutissent Bolton et Zwick à l'issue de leur étude sur le jeu de l'impunité montrent que les hypothèses de l'altruisme et de l'équité ne sont pas les meilleures candidates pour décrire l'attention à l'autre (Bolton et Zwick, 1995). Concernant le comportement de A, les résultats obtenus par Bolton et Zwick montrent que les joueurs A choisissent la proposition inégalitaire dans $98 \%$ des cas observés. Les rares choix de partage égalitaires sont observés en début de session et disparaissent après deux répétitions du jeu. Les participants A choisissent alors systématiquement les partages leur permettant de maximiser leur gain individuel et cela quel que soit l'écart entre leur propre gain et celui de B. Quant au comportement des joueurs B, Bolton et Zwick n'observent aucun rejet de proposition dans le jeu de l'impunité. Ce résultat, lui aussi, remet en cause l'hypothèse de l'équité. En effet, cette hypothèse dit que le rejet par B de la proposition est l'expression d'un désaccord face à une solution inéquitable. Dans le jeu de l'impunité, B devrait donc refuser le partage proposé quand celui-ci conduit à un partage du profit joint inégalitaire. En revanche l'hypothèse de la réciprocité dit que le rejet de la proposition a pour objectif de sanctionner le comportement de A. Les résultats obtenus pour le jeu de l'impunité montrent que cette seconde hypothèse s'avère être la plus pertinente. Lorsque le rejet de la proposition est sans effet sur le gain de A, aucun des sujets B ne refuse le partage inégalitaire même lorsque la part qui lui est attribuée est minime.

Les résultats obtenus pour le jeu de l'impunité montrent que les individus sont sensibles au comportement inéquitable de l'autre quand ce comportement s'exerce en sa défaveur. Si le négociateur se considère "agressé » dans ses intérêts par le comportement de l'autre et s'il a les moyens de répliquer à cette agression, il accepte alors de sacrifier une partie de ses gains monétaires pour 
sanctionner le comportement inéquitable. L'expérience sur le jeu de l'investissement permet d'observer que la réciprocité s'exerce aussi dans le sens inverse. Lorsqu'un agent considère que l'autre a agi à son endroit d'une manière qui dépasse les exigences des normes d'équité, il sera alors prêt à sacrifier une partie de ses gains monétaires pour récompenser la bienveillance de l'autre.

Dans le jeu de l'investissement, deux joueurs, A et B reçoivent chacun une dotation initiale d'un même montant. A peut choisir d'envoyer une part de sa dotation initiale à $\mathrm{B}$. Ce montant est multiplié par trois avant de parvenir à B. L'investissement de A crée donc un surplus. Dans la seconde phase du jeu, B décide quelle est la part du montant reçu, le profit joint, qu'il souhaite conserver et quelle est celle qu'il souhaite renvoyer à $\mathrm{A}$. Cette seconde phase de jeu correspond à un jeu du dictateur qui a fait l'objet de nombreuses études expérimentales ${ }^{7}$. Les prédictions de la théorie pour ce jeu sont évidentes. B a intérêt à s'approprier l'intégralité du profit joint. Anticipant le comportement de B, A a intérêt à ne rien investir. Comme pour le jeu de l'ultimatum, les résultats d'expériences réalisées sur la base du jeu de l'investissement ne s'accordent pas avec les prédictions de la théorie. Ainsi l'expérience conduite par Berg, Dickhaut et McCabe (1993) débouche sur les résultats suivants : A effectue des investissements qui sont manifestement différents de zéro ; B accorde à A une part du profit joint qui est nettement différente de zéro. Contrairement à ce qui est observé dans les expériences dédiées au jeu du dictateur simple, il apparait qu'un grand nombre de sujets B soit prêt à sacrifier une partie de son gain monétaire pour accroître celui de A. Ce comportement est en accord avec l'hypothèse de la réciprocité : en acceptant d'investir, A s'expose volontairement à l'opportunisme de B. La part du profit joint que lui accorde en retour B peut être considérée comme la contrepartie de cette confiance. De la sorte, il semblerait que les agents soient prêts à sacrifier une partie de leur gain monétaire pour récompenser la bienveillance de l'autre. Tout comme dans le jeu de l’ultimatum, la réciprocité apparaît ici comme la logique sous-jacente aux préférences sociales.

\section{CONCLUSION}

L'économie expérimentale constitue un outil privilégié pour étudier l'importance et la nature des préférences sociales en situation d'interaction stratégique. Les résultats des expériences présentées dans cet article montre que les individus ne sont pas uniquement motivés par l'importance de leur gain individuel, des préférences sociales interviennent dans la sélection des stratégies de négociation. Ces préférences sociales ne peuvent pas être interprétées comme une simple recherche de solutions «socialement correctes ». Les normes de justice et d'équité ont une place centrale dans l'évaluation par les agents des issues de la négociation. Cependant, les solutions équitables ne sont pas recherchées pour elles-mêmes; l'équité s'intègre dans une analyse du jeu qui reste stratégique. La réciprocité semble constituer l'élément central des préférences sociales des négociateurs. Chaque agent évalue le comportement de l'autre à son égard sur la base d'un ensemble de normes qui lui sont propres. De

\footnotetext{
${ }^{7}$ Voir par exemple (Hoffman et al., 1994).
} 
cette évaluation naît des sentiments de bienveillance ou de malveillance vis-à-vis de l'autre qui se traduisent dans la sélection d'une stratégie de négociation par l'agent.

L'introduction de préférences sociales dans le jeu de l'ultimatum pose un problème supplémentaire généré par l'incomplétude de l'information quant à la nature et l'importance des préférences sociales pour chacun des négociateurs. Pour le joueur A, il est ainsi nécessaire d'anticiper le comportement de $\mathrm{B}$ et donc de conjecturer la nature des motivations de ce dernier pour éviter le conflit. Dans le cadre d'une expérience, en l'absence d'une connaissance commune au sein de la cohorte quant à la nature et à l'importance des préférences de sociales de chacun des sujets, les négociateurs font face à un problème de coordination. Chacun cherchera à estimer les motivations de l'autre sur la base de ses propres motivations et de ses croyances.

\section{Références}

Berg John, John Dickhaut and Kevin McCabe. 1993. "Trust, Reciprocity, and Social History». Games and Economic Behavior, vol.10, p.122-142.

Binmore K., A. Shaked and J. Sutton. 1985. «Testing Noncooperative Bargaining Theory: A Preliminary Study ». The American Economic Review, vol.75 n5, p.1178-1180.

Bolton Gary E. 1991. «A Comparative Model of Bargaining: Theory and Evidence ». The American Economic Review, vol.81 n5, p.1097-1136.

Bolton Gary E. and Michael M. Zwick. 1995. «Anonymity Versus Punishment in Ultimatum Bargaining ». Games and Economic Behavior, vol.10 n¹, p.95-121.

Camerer Colin F. 2003. Behavioral Game Theory: Experiments in Strategic Interaction. Princeton: Princeton University Press et New York : Russell Sage Foundation.

Dufwenberg Martin and Georg Kirchsteiger. 2004. «A Theory of Sequential Reciprocity ». Games and Economic Behavior, vol.47 n², p.268-298.

Fehr Ernst and Klaus Schmidt. 1999. "A Theory of Fairness, Competition, and Cooperation». Quarterly Journal of Economics, vol.114 August, p.769-816.

Güth Werner. 1995. «On Ultimatum Bargaining Experiments: A Personal Review ». Journal of Economic Behavior and Organization, n²7, p.329-344.

Güth Werner, R. Schmittberger and B. Schwarz. 1982. «An Experimental Analysis of Ultimatum Bargaining ». Journal of Economic Behavior \& Organization, n³, p.367-388.

Hoffman Elizabeth, Kevin McCabe, Keith Shachat and Vernon Smith. 1994. «Preferences, Property Rights, and Anonymity in Bargaining Games ». Games and Economic Behavior, vol.7 n³, p.346-380.

Hoffman Elizabeth, Kevin A. McCabe, and Vernon L. Smith. 1996. «On Expectations and the Monetary Stakes in Ultimatum Games ». International Journal of Game Theory, vol.25 n³, p.289-301.

Meidinger Claude, Stéphane Robin et Bernard Ruffieux. 1999. «Confiance, Réciprocité et "Cheap Talk". Revue Economique, vol.50 n 1, p.5-44.

Ochs Jack and Alvin E. Roth. 1989. "An Experimental Study of Sequential Bargaining ». The American Economic Review, vol.79 n³, p.355-384. 
Rabin Matthew. 1993. "Incorporating Fairness into Game Theory and Economics ». American Economic Review, vol.83 December, p.1281-1302.

Roth Alvin E. 1995. «Bargaining Experiments ». In J.H. Kagel and A.E. Roth (eds), Handbook of Experimental Economics, p. 253-341. Princeton : Princeton University Press, 1995.

Roth Alvin E., Vesna Prasnikar, Masahiro Okuno Fujiwara and Shmuel Zamir. 1991. «Bargaining and Market Behavior in Jerusalem, Ljubjana, Pittsburg, and Tokyo: An Experimental Study ». American Economic Review, vol.81 n5, p.1068-1095.

Thaler Richard H. 1988. "Anomalies: The Ultimatum Game». The Journal of Economic Perspectives, vol. 2 n4, p.195-206. 